\title{
Use of clinical practice guidelines
}

\author{
Ben YF Fong *, MPH(Syd), FHKAM (Community Medicine) \\ Division of Science, Engineering and Health Studies, College of Professional and Continuing Education, The Hong Kong Polytechnic \\ University, Hong Kong \\ *Corresponding author: byffong@gmail.com \\ Hong Kong Med J 2020;26:367-9 \\ https://doi.org/10.12809/hkmj205106
}

In society, guidelines shape the behaviour and steer the activities of people and organisations in all aspects of daily routine. In the clinical setting, professional care and services are often dictated by clinical practice guidelines (CPGs) on topics from screening, assessment, and diagnosis to management of common and specific conditions. There are also CPGs for unusual emerging pandemics: in the case of coronavirus disease 2019 (COVID-19) the World Health Organization has published extensive advice. ${ }^{1}$

According to the National Institute of Health and Care Excellence of the United Kingdom, CPGs contain evidence-based recommendations on the ways healthcare professionals should care for people with defined conditions. Such recommendations are derived from the best available evidence. In addition, CPGs are also of importance to health services operators and managers as such guidelines are essential for quality care. ${ }^{2}$

Effective CPGs are derived from evidencebased medicine, and should be built on the best available published research findings and experience gained from clinical practice. To achieve the optimal usage of CPGs, healthcare professionals should understand the principles of development and evaluation of the guideline. The AGREE II guidelines and associated reporting checklist is considered the international gold standard for developing and evaluating CPGs. ${ }^{3}$ Quality, relevance, and strength of the best available evidence are examined in detail with a multidisciplinary approach when making recommendations for clinical management. Ideally, CPGs are embedded with flexibility and adaptability to allow for a wide dissemination and adoption. In addition, potential economic implications should not be overlooked. ${ }^{4}$ Practically, CPGs should be subject to regular structured evaluation and revision to encompass the latest state-of-the-art of clinical practice, new research evidence, current medical advancement, and changed patient values.

Topics of CPGs are often selected on the basis of priorities in quality improvement opportunities in medical practice because physicians are expected to provide their patients a certain standard of care. In a review of CPGs, consensus statements, and position statements from various specialties, the authors found that to achieve the optimal benefits from providing the best possible and quality care to the community, topics of CPGs should cover common conditions that doctors encounter regularly. ${ }^{5}$ In this issue of the Hong Kong Medical Journal, Chan et al from the Hong Kong College of Physicians, representing sub-specialties in Cardiology, Nephrology, Geriatric Medicine, Neurology and Endocrinology, review two recent CPGs from America and Europe on hypertension, both of which have adopted a risk-based approach to treatment. ${ }^{6}$ On the basis of these guidelines, the authors have developed a Position Statement on the classification of blood pressure, measurement of blood pressure, initiation of medications, treatment targets and strategies, together with particular considerations for geriatric, renal, and diabetic patients. ${ }^{6}$ In general, the authors concur with the 2018 European Society of Cardiology/European Society of Hypertension guideline, and also note that the reviewed guidelines have helped to improve public awareness of hypertension and the importance of lifestyle changes in managing hypertension.

Physicians use CPGs when making clinical decisions, often with discussion with the patient in some settings, about the appropriate care and management of specific conditions or diseases. Reviewing CPGs published in the Hong Kong Medical Journal, some have provided guidance on disease screening and prevention, ${ }^{7,8}$ whereas others have offered a broad range of clinical topics from management of common clinical complaints to controversial issues based on the most updated evidence. ${ }^{9-13}$ Guidelines include concise recommendations and instructions for diagnosis, tests, treatment options, drug therapy, management algorithms that may direct the choice of medical, and surgical or other clinical services. ${ }^{14}$ Because CPGs are never perfect and evidence on their effectiveness is incomplete, ${ }^{14}$ when making clinical decisions, doctors also consider their experience and knowledge through years of practice and insights gained from the many cases encountered and managed previously. Therefore, most doctors have developed an individual approach to patient management without consciously thinking about CPGs. However, CPGs are useful when dealing with uncommon conditions or diseases unfamiliar to the practitioner, as well as in controversial clinical situation. Under such circumstances, there is a high 
level of uncertainty as to the outcomes of clinical courses being available or considered, such as the current COVID-19 pandemic.

Adherence to CPGs in clinical practice is not mandatory. However, the Medical Council of Hong Kong has some guidelines to doctors in the Code of Professional Conduct, "the little red book", with the intention to promote good clinical practice. ${ }^{15}$ There are guidelines on the proper prescription and dispensing of dangerous drugs, ethics (covering communication and dissemination of information to the public and patients), adoption of new medical procedures and human reproductive technology, as well as on practice management including signboards, service information notices, and doctors directory. In addition, legal considerations are practical issues when CPGs are not fully followed or when treatment recommended in CPGs is not offered to the patient. Following the advice found in CPGs may provide a means of protection to the doctor, as CPGs prescribe reasonable conduct expected of medical practitioners who are also expected to provide sufficient information to the patient, particularly about risks involved in a clinical decision.

Some CPGs contain definitive recommendations whereas others are more general, to allow for the clinical judgement of the practitioner, and therefore carry a smaller risk of liability. Generally, groups who develop CPGs, such as members of a working group, are not usually held liable for the application of CPGs. Furthermore, use of CPGs as evidence in court depends on how the guidelines are developed and whether they are up to date. Good CPGs should have a strong research evidence base, should have undergone independent review, should have built-in flexibility for adoption in different clinical situation, and should carry an expiry date. ${ }^{4}$ Practitioners should have adequate understanding of the salient points in CPGs to fully exploit the guidelines in clinical settings.

The purpose of CPGs is to improve the health and condition of patients, not only as individuals but also as members of the community, particularly when society is facing escalating costs of health services and medical technologies, ageing populations, increasing demands and expectations of users, inconsistent service quality of providers, and even inappropriate care. Use of CPGs by physicians in clinical practice can lead to more structured and consistent decisions and care delivery in a more objective manner, supported by the embedded evidence. The Primary Healthcare Office of the Hong Kong SAR Government has published four reference frameworks on disease management of hypertension and diabetes, and on preventive care for children and elderly patients. The frameworks aim to facilitate family doctors to provide continuing comprehensive and evidence-based care in the community. ${ }^{16}$ In the code of practice to private hospitals and clinics, the Department of Health demands that these institutions comply with guidelines and standards issued by professional and government bodies, and that CPGs must be easily accessible and available to staff for their reference. ${ }^{17}$

Doctors should use appropriate CPGs in their daily practice for the benefit and better quality of life of their patients. However, CPGs must not be regarded as the absolute consensus recommendations with answers to all clinical situations. Intrinsic shortcomings in the development of guidelines are not uncommon. Patients are not all the same; they vary in personal characteristics and responses to treatments. Moreover, doctors differ in their clinical judgement. Use of CPGs by physicians is still very much the core of medicine, being both an art and a science.

\section{Author contributions}

The author contributed to the editorial, approved the final version for publication, and takes responsibility for its accuracy and integrity.

\section{Conflicts of interest}

The author has disclosed no conflicts of interest.

\section{References}

1. World Health Organization. Clinical management of COVID-19-interim guidance. Available from: https:// www.who.int/publications/i/item/clinical-managementof-covid-19. Accessed 5 Sep 2020.

2. National Institute for Health and Care Excellence. What is a NICE clinical guideline? Available from: https://www. nice.org.uk/process/pmg6/resources/how-nice-clinicalguidelines-are-developed-an-overview-for-stakeholdersthe-public-and-the-nhs-2549708893/chapter/niceclinical-guidelines\#what-is-a-nice-clinical-guideline. Accessed 5 Sep 2020.

3. Brouwers MC, Kerkvliet K, Spithoff K, AGREE Next Steps Consortium. The AGREE Reporting Checklist: a tool to improve reporting of clinical practice guidelines. BMJ 2016;352:i1152.

4. National Health and Medical Research Council. A Guide to the Development, Implementation and Evaluation of Clinical Practice Guidelines. Australia: NHMRC; 1999.

5. Chong CC. Pros and cons of clinical practice based on guidelines. Hong Kong Med J 2018;24:440-1.

6. Chan KK, Szeto CC, Lum CC, et al. Hong Kong College of Physicians Position Statement and Recommendations on the 2017 American College of Cardiology/American Heart Association and the 2018 European Society of Cardiology/ European Society of Hypertension Guidelines for the Management of Arterial Hypertension. Hong Kong Med J 2020;26:432-7.

7. Cancer Expert Working Group on Cancer Prevention and Screening. Recommendations on prevention and screening for colorectal cancer in Hong Kong. Hong Kong Med J 2018;24:521-6. 
8. Cancer Expert Working Group on Cancer Prevention and Screening. Recommendations on prevention and screening for breast cancer in Hong Kong. Hong Kong Med J 2018;24:298-306.

9. Tomlinson B, Chan JC, Chan WB, et al. Guidance on the management of familial hypercholesterolaemia in Hong Kong: an expert panel consensus viewpoint. Hong Kong Med J 2018;24:408-15.

10. Mak LY, Lau CW, Hui YT, et al. Joint recommendations on management of anaemia in patients with gastrointestinal bleeding in Hong Kong. Hong Kong Med J 2018;24:41622 .

11. Lim MK, Ha SC, Luk KH, et al. Update on the Hong Kong Reference Framework for Hypertension Care for Adults in Primary Care Settings-review of evidence on the definition of high blood pressure and goal of therapy. Hong Kong Med J 2019;25:64-7.

12. The Advisory Group on Antibiotic Stewardship Programme in Primary Care. Antibiotic management of acute pharyngitis in primary care. Hong Kong Med J
2019;25:58-63.

13. Wu JC, Chan AO, Cheung TK, et al. Consensus statements on diagnosis and management of chronic idiopathic constipation in adults in Hong Kong. Hong Kong Med J 2019;25:142-8.

14. Woolf SH, Grol R, Eccles M, Grimshaw J. Clinical guidelines: potential benefits, limitations, and harms of clinical guidelines. BMJ 1999;318:527-30.

15. Medical Council of Hong Kong. Code of professional conduct. Available from: https://www.mchk.org.hk/ english/code/files/Code_of_Professional_Conduct_2016. pdf. Accessed 5 Sep 2020.

16. Primary Healthcare Office, Food and Health Bureau, Hong Kong SAR Government. Reference frameworks. Available from: https://www.fhb.gov.hk/pho/english/initiatives/ frameworks.html. Accessed 5 Sep 2020.

17. Office for Regulation of Private Healthcare Facilities, Department of Health, Hong Kong SAR Government. Regulatory regime. Available from: https:/www.orphf.gov. hk/en/regulatory_regime/index. Accessed 5 Sep 2020. 